\title{
CORRECTION
}

\section{A Note On Necessary Best Estimator Of Order Two}

\section{By S.G. Prabhu-Ajgaonkar, Aurangabad 431004}

Metrika, Volume 31, 1984, page 1-4.

Equations (14) to (18) are incorrect. The corrected equation (14) is as follows:

$$
\begin{aligned}
E\left(\frac{1}{\pi_{\lambda} \pi_{\lambda^{\prime}}}\right) & =\sum_{s \supset \lambda \lambda^{\prime}} \frac{p(s)}{\pi_{\lambda} \pi_{\lambda^{\prime}}} \\
& =\frac{\pi_{\lambda \lambda^{\prime}}}{\pi_{\lambda} \pi_{\lambda^{\prime}}}
\end{aligned}
$$

On the same lines, let

$$
\begin{aligned}
& E\left(\frac{{ }^{e s} \lambda}{\pi_{\lambda^{\prime}}}\right)=E_{1} \\
& E \frac{e s \lambda_{\lambda^{\prime}}}{\pi_{\lambda}}=E_{2}
\end{aligned}
$$

Now equation (17) changes to

$$
E\left(\beta s \lambda \beta s \lambda^{\prime}\right)=\frac{{ }^{\pi} \lambda \lambda^{\prime}}{\pi_{\lambda} \pi_{\lambda^{\prime}}}+E_{1}+E_{2}+E\left(e_{s \lambda} e_{s \lambda^{\prime}}\right)
$$

Subsequently equation (18) changes to

$$
\begin{aligned}
Q^{\prime} & =\operatorname{Var}(t)-\operatorname{Var}\left(t_{H T}\right) \\
& =\sum_{\lambda=1}^{N} E\left(e_{s \lambda}^{2}\right) Y_{\lambda}^{2} \\
& +\sum_{\lambda \neq \lambda^{\prime}=1}^{N}\left[E_{1}+E_{2}+E\left(e_{s \lambda} e_{s \lambda^{\prime}}\right)\right] Y_{\lambda} Y_{\lambda^{\prime}}
\end{aligned}
$$

The Horvitz and Thompson estimator is the necessary best estimator of order two if for $\lambda \neq \lambda^{\prime}$

$$
E\left(e s_{\lambda}^{2}\right) E\left(s_{s \lambda^{\prime}}^{2}\right)-\left[E_{1}+E_{2}+E\left(e_{s \lambda} e_{s \lambda^{\prime}}\right)\right]^{2}
$$

is non-negative.

Consider the restricted class of unbiased estimators where for all $s$,

$$
\beta_{s \lambda}=\left[\begin{array}{ll}
\beta_{s \lambda} & \lambda \neq \lambda^{\prime}=1,2, \ldots, N . \\
\frac{1}{\pi_{\lambda^{\prime}}} & \text { for } \lambda=\lambda^{\prime}
\end{array}\right.
$$

For these estimators $e_{s \lambda^{\prime}}=0$ for all $s$, and the above expression reduces to

$$
-E_{1}^{2}
$$

which is negative.

This establishes the result that the necessary best estimator of order two does not exist in Lu. 